\title{
Comorbidades e sinais de alerta da pneumonia adquirida na comunidade: análise de pacientes com mais de 50 anos
}

\author{
Comorbidities and warning signals of community acquired pneumonia: \\ analysis of patients over 50 years old
}

Rodrigo Castro, Rodrigo Dal Moro Amarante, Maria Cecília de Toledo Damasceno, André Valente Lage, Gustavo Latorre Samençatti, Carla Komon de Souza

\section{Resumo}

Introdução: a pneumonia adquirida na comunidade (PAC) é a maior causa de morte por doenças infecciosas no mundo, principalmente em idosos. São Caetano do Sul, São Paulo, demonstra um aumento de população idosa (28\%), devido ao aumento da expectativa de vida. Assim, o número de casos em pacientes $\geq 50$ anos aumentou drasticamente. Dos 567 casos notificados em São Caetano Sul, de maio a dezembro de 2005 e de março a setembro de 2006, 70\% correspondiam a idosos. Objetivo: analisar as comorbidades relacionadas à PAC em pacientes $\geq 50$ anos e os sinais de alerta que estes apresentam no início do tratamento ambulatorial em São Caetano do Sul. Casuística e métodos: foram analisados 41 casos de PAC em pacientes $\geq 50$ anos que apresentavam o quadro clínico compatível com infecção respiratória aguda, achado de infiltrado pulmonar novo ao raio $\mathrm{X}$ de tórax, associado à pelo menos um dos itens: desidratação, febre $\geq 38^{\circ} \mathrm{C}$ e tosse produtiva. Os critérios de exclusão foram a presença de doenças pulmonares e portadores de HIV. Resultados e conclusões: analisou-se o quadro de PAC em 41 pacientes com $\geq 50$ anos, com sinais e sintomas característicos aos exames no Pronto Socorro. Devido à particularidade da amostra estudada, não se podem generalizar os resultados. Houve prevalência do sexo feminino, sendo a idade média dos pacientes de 69 anos Das comorbidades analisadas, a insuficiência cardíaca congestiva teve maior prevalência (50\%), seguida pela insuficiência renal (25\%). Dos pacientes analisados, 24\% apresentavam uréia > $40 \mathrm{mg} / \mathrm{dL}$ e 18\% apresentavam taquipnéia.

Palavras-Chave: Pneumonia adquirida na comunidade; comorbidade; sinais de alerta; São Caetano do Sul; idoso.

\section{Abstract}

Introduction: the Community acquired pneumonia (CAP) is the major cause of death by infectious diseases in the world, especially among the elderly. Sao Caetano do Sul, São Paulo, shows a growth of the elderly population (28\%), due to the rise of the life expectancy. Therefore, the number of cases in patients older than 50 years old rose drastically. From the 567 cases related in São Caetano do Sul, from May to December of 2005 and from March to September of 2006, 70\% were related to elders. Objective: analyze the comorbidities related to CAP in patients older than 50 years, and the alert signs shown by them in the beginning of the ambulatory treatment in São Caetano do Sul. Subjectives and methods: 41 cases of CAP were analyzed in patients older than 50 years which presented clinical situation compatible with deep breathing infection, new pulmonary infiltrate discovered in the X-Ray exam of the chest, which is related to at least one of the following items: dehydration, fever and productive cough. The exclusion criteria were the diagnosis of lung diseases and HIV. Results and conclusions: the CAP scenes of 41 patients older then 50 years were analyzed, with signs and symptoms that are characteristics of the Emergency Medical Services exams. Due to the particularity of the sample studied, the result cannot be generalized. Females prevailed; the average age of the patients was 69 years. From all the comorbidities analyzed, the congestive cardiac insufficiency prevailed at most ( $50 \%)$, followed by the renal insufficiency (25\%). Among the patients analyzed, $24 \%$ presented urea $>40 \mathrm{mg} / \mathrm{dL}$ and $18 \%$ presented shortpnea.

Keywords: Community-acquired pneumonia; comorbidity; alert signs; Sao Caetano do Sul; elderly.

Recebido: 24/10/2007

Revisado: $12 / 05 / 2008$

Aprovado: 13/06/2008

Disciplina de Medicina de Urgência da Faculdade de Medicina do ABC, Santo André, São Paulo, Brasil.

Endereço para correspondência: Rua Maranjaí, 459 - Vila Monumento - São Paulo, SP - e-mail: ro_castro@terra.com.br 


\section{Introdução}

A pneumonia adquirida na comunidade (PAC) é uma doença que acomete a população fora do ambiente hospitalar ou pacientes nas primeiras 48 horas de internação. Vários agentes etiológicos a causam, como o Streptococcus pneumoniae, Staphylococcus aureus, bacilos Gramnegativos, Mycoplasma pneumoniae, influenza vírus A e $\mathrm{B}^{1}$.

A maioria dos estudos a respeito da etiologia das PACs foi feita em pacientes internados, mas, nos últimos anos, estudos realizados em pacientes não internados foram publicados. Nestes, no total de 1.091 casos foram avaliados, sendo a média ponderal das etiologias: Streptococcus pneumoniae, 22\%; Mycoplasma pneumoniae, 18\%; Chlamydia pneumoniae, 16\%; vírus respiratório, 10\%; Haemophilus influenzae, 4\% e Legionella $s p$ foi rara. Em sete estudos de pacientes com PAC internados, foram calculadas também as médias das diversas etiologias, sendo mais prevalente o Streptococcus pneumoniae, 17\%; Mycoplasma pneumoniae, 6\%; Chlamydia pneumoniae, 6\%; Legionella sp, 4\% e vírus respiratórios, $7 \%{ }^{2}$.

A Pneumonia adquirida na comunidade continua sendo uma das maiores causas de morte por doenças infecciosas no mundo, apesar de todo o avanço na área médica e social, no decorrer do século e da disponibilidade de novos antibióticos ${ }^{3}$. Nos Estados Unidos, a PAC representa a sexta causa de morte ${ }^{4}$ e no Brasil, a quinta ${ }^{5}$. A gravidade dessa doença pode variar de uma infecção benigna em adultos jovens até casos que acarretam graves morbidades e mortalidades nos pacientes idosos e imunocomprometidos. O surgimento da pneumonia indica anormalidades das defesas dos hospedeiros, presença de um microorganismo particularmente virulento ou ocorrência de inoculações maciças ${ }^{1}$.

Mesmo com o avanço obtido nas técnicas de diagnóstico, estimase que cerca de $50 \%$ dos casos ainda permaneçam sem etiologia definida. Aproximadamente $80 \%$ dos adultos com PAC são tratados ambulatorialmente, mantendo a taxa de mortalidade baixa em torno de 1 a $5 \%{ }^{4}$. Entretanto, nos pacientes que requerem hospitalização, essa taxa aumenta de forma significativa, particularmente se o paciente necessita de admissão em uma unidade de tratamento intensivo ${ }^{6-9}$. A definição do local de tratamento constitui uma etapa fundamental na terapêutica, que tem grande repercussão em seus resultados e custos. Em virtude disso, criaram-se critérios auxiliares no reconhecimento de pacientes com PAC de menor risco, passíveis de receberem tratamento em nível ambulatorial ${ }^{9,10}$, tendo como objetivo a redução de custos nos resultados terapêuticos apresentados.

$\mathrm{O}$ aumento na expectativa de vida, em conseqüência da melhoria da qualidade da assistência médica, do saneamento básico e a redução da fecundidade, são fatores responsáveis pela modificação da pirâmide etária, no sentido de um grande aumento da população idosa que é mais vulnerável ao desenvolvimento de pneumonias, devido às alterações provocadas pela própria senescência e por patologias associadas ${ }^{11}$.

São Caetano do Sul é uma cidade que demonstra esta alteração na pirâmide etária, apresentando um total de $36 \%$ de população com mais de 50 anos $^{12}$. Com isso, o número de casos de pneumonia, em pacientes com mais de 50 anos, vem aumentando drasticamente, obrigando mudanças na estrutura hospitalar para melhor atendê-los. Dos 567 casos de pneumonia notificados em São Caetano do Sul, no período de maio a dezembro de 2005 e março a setembro de 2006, incluindo os pacientes tratados ambulatorialmente, $70 \%$ correspondiam a infecções em idosos 5 .

A incidência da PAC é crescente no mundo e, em parte, deve-se ao aumento de alguns fatores como: idade crescente da população e aumentos de comorbidades, como doença pulmonar obstrutiva crônica (DPOC), acidente vascular cerebral (AVC), neoplasias, insuficiência renal, entre outras. Além disso, a PAC apresenta pior prognóstico quando tais comorbidades estão presentes ${ }^{13}$. No Brasil, a pneumonias adquirida na comunidade é a segunda causa de internação, sendo a maior proporção nos meses de inverno ${ }^{5}$.

O objetivo deste trabalho foi descrever as comorbidades associadas à pneumonia em 41 pacientes com 50 anos ou mais que deram entrada no Pronto Socorro Municipal de São Caetano do Sul.

\section{Casuística e métodos}

Este estudo descreveu de forma retrospectiva 41 casos de PAC em pacientes com mais de 50 anos, que deram entrada no Prontosocorro Maria Braido, em São Caetano do Sul, no período de maio a dezembro de 2005 e março a setembro de 2006. Os pacientes inicialmente eram avaliados por um médico e, uma vez confirmada a suspeita de PAC, os mesmos eram submetidos a um protocolo de avaliação de gravidade que utilizava critérios Patient Outcomes Research Team (PORT). Os critérios PORT consideram avaliações como a medição da saturação de oxigênio (oximetria de pulso), a gasometria arterial, caso houvesse hipoxemia, a insuficiência respiratória, a ausência ou presença de insuficiência cardíaca congestiva (ICC), a presença de neoplasias, de doenças hepáticas, de insuficiência renal ou de AVC. Os dados coletados foram registrados em protocolo de atendimento médico padronizado. Todos os pacientes com suspeita de PAC foram submetidos ao um exame de radiologia simples de tórax. Apesar de não haver padrões radiológicos diagnosticados, alguns achados podem sugerir determinadas etiologias ou detectar alternativas diagnósticas não-infecciosa.

Os critérios de inclusão dos pacientes foram o de quadro clínico compatível com infecção respiratória aguda, o achado de um infiltrado pulmonar novo à radiografia do tórax associada à pelo menos um dos seguintes itens:

- desidratação;

- febre $\geq 38^{\circ} \mathrm{C}$;

- tosse produtiva.

Além disso, todos os pacientes no referente estudo, apresentavam idade superior a 50 anos. 
Tabela 1 - Relação do sexo com o número de PAC entre maio a dezembro de 2005 e março a setembro de 2006

\begin{tabular}{lcc}
\hline Ano & \multicolumn{2}{c}{$2005 / 2006$} \\
\hline Sexo & Número & Porcentagem \\
\hline Masculino & 15 & 36,5 \\
Feminino & 26 & 63,5 \\
Total & 41 & 100,0 \\
\hline
\end{tabular}

Tabela 2 - Relação das comorbidades associadas à pneumonia nos períodos de maio a dezembro de 2005 e março a setembro de 2006

\begin{tabular}{lcc}
\hline Ano & \multicolumn{2}{c}{$2005 / 2006$} \\
\hline Comorbidade & Número & Porcentagem \\
\hline Insuficiência renal & 6 & 25 \\
Insuficiência cardíaca congestiva & 12 & 50 \\
Acidente vascular cerebral & 4 & 16,6 \\
Hepatopatia crônica & 1 & 4,2 \\
Doença pulmonar obstrutiva crônica & 1 & 4,2 \\
Neoplasias & 0 & 0,0 \\
Total & 24 & 100,0 \\
\hline
\end{tabular}

Tabela 3 - Relação dos sinais de alerta relacionados à pneumonia no período de maio a dezembro de 2005 e março a setembro de 2006

\begin{tabular}{lcc}
\hline Ano & \multicolumn{2}{c}{$2005 / 2006$} \\
\hline Sinal de alerta & Número & Porcentagem \\
\hline Confusão mental aguda & 4 & 5,2 \\
PAS $<90 \mathrm{mmHg} / \mathrm{PAD}>60 \mathrm{mmHg}$ & 5 & 6,5 \\
Temperatura axilar $<35{ }^{\circ} \mathrm{C}$ ou $>40{ }^{\circ} \mathrm{C}$ & 2 & 2,6 \\
Taquicardia & 4 & 5,2 \\
Taquipnéia $>30 \mathrm{rpm}$ & 14 & 18,2 \\
$\mathrm{pH}$ arterial $<7,35$ & 8 & 10,4 \\
Uréia $>40 \mathrm{mg} / \mathrm{dL}$ & 18 & 23,3 \\
Glicose $>250 \mathrm{mg} / \mathrm{dL}$ & 3 & 3,8 \\
Sódio $<130 \mathrm{mEq} / \mathrm{L}$ & 2 & 2,6 \\
Hemoglobina $<9 \mathrm{~g} / \mathrm{dL}$ & 1 & 1,4 \\
Derrame pleural & 6 & 7,8 \\
$\mathrm{pO}_{2}<60$ ou saturação $<90 \%$ & 10 & 13,0 \\
Total & 77 & 100,0 \\
\hline
\end{tabular}

Os critérios de exclusão foram diagnósticos alternativos como edema pulmonar, tromboembolismo pulmonar, tuberculose pulmonar, pneumonia nosocomial e pacientes infectados com HIV.

As comorbidades analisadas foram: insuficiência renal, insuficiência cardíaca congestiva (ICC), AVC, hepatopatia crônica, DPOC e neoplasias torácicas. Foram também observados os sinais de alerta que estes pacientes apresentavam no início da avaliação clínica: confusão mental, taquipnéia, pressão arterial sistólica menor de $90 \mathrm{mmHg}$ e pressão arterial diastólica menor que $60 \mathrm{mmHg}$, temperatura axilar superior a $38^{\circ} \mathrm{C}$, pulso superior a $125 \mathrm{bpm}, \mathrm{pH}$ arterial menor que 7,25 , uréia maior que $40 \mathrm{mg} / \mathrm{dL}$, glicose superior a $250 \mathrm{mg} / \mathrm{dL}$, sódio inferior a $130 \mathrm{mEq} / \mathrm{L}$, hemoglobina inferior a 9 $\mathrm{g} / \mathrm{dL}$, presença de derrame pleural e saturação de oxigênio inferior a $90 \%$.

\section{Resultados}

Foram avaliados 46 pacientes com mais de 50 anos, atendidos com o diagnóstico de PAC no Pronto Socorro Municipal de São Caetano do Sul. Destes, cinco foram excluídos por não apresentarem todos os critérios de inclusão.

A média de idade \pm desvio padrão dos pacientes foi de $69 \pm 14,8$ anos. As mulheres representaram $66 \%$ dos casos notificados de PAC, demonstrando uma maior prevalência da doença no sexo feminino, durante os dois períodos analisados (Tabela 1). Uma possível explicação pela maior prevalência de mulheres pode estar relacionada à expectativa de vida das mulheres, que é mais alta do que a dos homens.

Todos os pacientes analisados apresentavam comorbidades associadas à pneumonia adquirida na comunidade. A insuficiência cardíaca congestiva demonstra maior prevalência, estando presente em $50 \%$ dos casos, seguida pela insuficiência renal com 25\% (Tabela 2).

Com relação aos sinais de alerta analisados, viu-se que $24 \%$ apresentavam uréia superior a $40 \mathrm{mg} / \mathrm{dL}$ e $18 \%$ apresentavam taquipnéia com mais de 30 respirações por minuto, quando chegaram ao Pronto Socorro (Tabela 3).

\section{Discussão}

O presente estudo descreveu os pacientes com mais de 50 anos portadores de PAC. Há poucos estudos nacionais e internacionais envolvendo este tipo de paciente. Os resultados obtidos não podem ser generalizados, devido às particularidades da amostra estudada. Em nenhum dos estudos analisados ${ }^{4,7-10}$ houve relação das comorbidades e dos sinais de alerta com a doença.

Conclui-se que os pacientes com PAC apresentam sinais e sintomas muito característicos na chegada do Pronto Atendimento. Nos 41 casos estudados, notou-se uma prevalência do sexo feminino, devido à maior expectativa de vida das mulheres em comparação com a dos homens.

Com base nos dados apresentados, pode-se concluir que as comorbidades mais freqüentemente encontradas são a insuficiência cardíaca congestiva, insuficiência renal e AVC. Estes dados não afastam o diagnóstico de outras comorbidades.

A uréia maior do que $40 \mathrm{mg} / \mathrm{dL}$, a taquipnéia e a saturação de oxigênio menor que $90 \%$ são os sinais de alerta mais evidentes nos pacientes com PAC. Muitos destes sinais se mostraram relacionados com as comorbidades. Portanto, podemos inquirir que muitos dos sinais de alerta ocorrem devido às complicações das comorbidades nos pacientes com PAC.

\section{Agradecimentos}

À Janeth Ferreira Lima, da Diretoria Clínica do Pronto Socorro Municipal de São Caetano do Sul.

À Regina Maria Zetone Grespan, Diretora de Saúde de São Caetano do Sul. 


\section{Referências}

1. Frisoli Jr A, Lopes AC, Amaral JLG, Blum VF, Ferraro JR. Emergências Manual de diagnóstico e tratamento. 2ª ed. São Paulo, Brasil: Sarvier; 2004. p. 461-83.

2. Pereira CAC, Rocha RT, Silva RF. Diretrizes para Pneumonias Adquiridas na Comunidade (PAC) em Adultos Imunocompetentes. J Bras Pneumol 2004:30(Supl. 4); S1-S24.

3. Almeida JR, Ferreira Filho OF. Pneumonias adquiridas na comunidade em pacientes idosos: aderência ao Consenso Brasileiro sobre Pneumonias. J Bras Pneumol 2004;30(3):229-36.

4. Niederman MS, Bass JB Jr, Campbell GD, Fein AM, Grossman RF, Mandell LA et al. Guidelines for the initial management of adults with community-acquired pneumonia: diagnosis, assessment of severity, and initial antimicrobial therapy. American Thoracic Society. Medical Section of the American Lung Association. Am Rev Respir Dis 1993;148(5):1418-26.

5. Brasil. Ministério da Saúde. Datasus. Disponível em: URL: http://www. datasus.gov.br. Acessado em 16 de janeiro de 2007.

6. Guidelines for the management of community-acquired pneumonia in adults admitted to hospital. British thoracic Society. Br J Hosp Med 1993;49(5):346-50.
7. Fine MJ, Smith MA, Carson CA, Mutha SS, Sankey SS, Weissfeld LA et al. Prognosis and outcomes of patients with community-acquired pneumonia. A meta-analysis. JAMA 1996;275(2):134-41.

8. Fang GD, Fine M, Orloff J, Arisumi D, Yu VL, Kapoor W et al. New and emerging etiologies for community-acquired pneumonia with implications for therapy. A prospective multicenter study of 359 cases. Medicine (Baltimore) 1990;69(5):307-16.

9. Bartlett JG, Dowell SF, Mandell LA, File Jr TM, Musher DM, Fine MJ. Practice guidelines for the management of community-acquired pneumonia in adults. Infectious Diseases Society of America. Clin Infect Dis 2000;31(2):347-82.

10. Fine MJ, Auble TE, Yealy DM, Hanusa BH, Weissfeld LA, Singer DE et al. A prediction rule to identify low-risk patients with community-acquired pneumonia. N Engl J Med 1997;336(4):243-50.

11. Schoueri JR, Ramos LR, Papaleo NM. Crescimento populacional: aspectos demográficos e sociais. In: Carvalho Filho ET, Papaleo NM. Geriatria: Fundamentos, Clínica e Terapêutica. $1^{a}$ ed. São Paulo: Atheneu: 2000, p. 9-29.

12. Brasil. IBGE. 2007. Disponível em: URL: http://www.ibge.gov.br. Acessado em 16 de janeiro de 2007.

13. Martins HS, Neto RAB, Neto AS, Velasco IT. Emergências Clínicas: Abordagem Prática. $2^{\mathrm{a}}$ ed. São Paulo: Manole: 2006, p. 461-83. 